\title{
Downregulation of microRNA-146a inhibits ovarian granulosa cell apoptosis by simultaneously targeting interleukin-1 receptor-associated kinase and tumor necrosis factor receptor-associated factor 6
}

\author{
XI CHEN, MINGXUAN XIE, DA LIU and KE SHI \\ Department of Geriatric Medicine, Xiangya Hospital, Central South University, Changsha, Hunan 410008, P.R. China
}

Received September 5, 2014; Accepted May 13, 2015

DOI: $10.3892 / \mathrm{mmr} .2015 .4036$

\begin{abstract}
Premature ovarian failure (POF), an ovarian disorder of multifactorial origin, is defined as the occurrence of amenorrhea, hypergonadotropism and hypoestrogenism in females $<40$ years old. Apoptosis of ovarian granulosa cells is important in POF and understanding the regulatory mechanism underlying ovarian granulosa cell apoptosis may be beneficial for the management of POF. Increasing evidence suggests that microRNAs (miRs) have a regulatory function in oocyte maturation and ovarian follicular development. In the present study, the expression of miR-146a in plasma and ovarian granulosa cells obtained from patients with POF, its effect on the apoptosis of ovarian granulosa cells and the possible underlying mechanisms were examined. The present study demonstrated that compared with the control groups, the expression of miR-146a in the plasma and in ovarian granulosa cells of patients with POF was significantly upregulated. Furthermore, it was found that miR-146a simultaneously targeted interleukin-1 receptor-associated kinase (IRAK1) and tumor necrosis factor receptor-associated factor 6 (TRAF6), which regulated the activity of nuclear factor- $\kappa \mathrm{B}$ and $\mathrm{I} \kappa \mathrm{B} \alpha$. In addition, the results demonstrated that inhibition of the caspase cascade by caspase inhibitors attenuated the effects of miR-146a on ovarian granulosa cell apoptosis. Taken together, these results suggest that miR-146a has an important promoting effect on the apoptosis of granulosa cells by targeting IRAK1 and TRAF6 via the caspase cascade pathway. These results may be useful for the management of POF.
\end{abstract}

Correspondence to: Professor Ke Shi, Department of Geriatric Medicine, Xiangya Hospital, Central South University, 87 Xiangya Road, Changsha, Hunan 410008, P.R. China

E-mail: csushike@163.com

Key words: premature ovarian failure, microRNAs, microRNA-146a, interleukin-1 receptor-associated kinase, tumor necrosis factor receptor-associated factor 6 , apoptosis

\section{Introduction}

Premature ovarian failure (POF), an ovarian disorder of multifactorial origin, is defined as the occurrence of amenorrhea, hypergonadotropism and hypoestrogenism in females $<40$ years old (1). POF results in fertility problems and leads to an increased incidence of cardiovascular disease, stroke and osteoporosis, a severe endocrine disorder (2). It has been reported that POF may result from either a reduced number of follicles formed during ovarian development or an increased rate of follicle loss (3). Previous studies have demonstrated that various factors, including androgens (4), can induce apoptosis in ovarian granulosa cells and result in follicle loss (5). Thus, the apoptosis of ovarian granulosa cells is important in POF and understanding the regulatory mechanism underlying ovarian granulosa cell apoptosis may be advantageous for the management of POF.

MicroRNAs (miRNAs), a class of gene negative regulators, are short ( $22 \mathrm{nt})$ noncoding RNAs. miRNAs repress the expression of target genes by degrading or destabilizing their mRNA in a sequence-specific manner (6). In this way, miRNAs affect various cellular processes, including cell proliferation, differentiation and apoptosis under normal and diseased conditions (7).

Increasing evidence suggests that miRNAs have a regulatory function in oocyte maturation and ovarian follicular development (8). Numerous differentially expressed miRNAs have been identified in ovary-associated diseases. Fiedler et al identified 13 differentially expressed miRNAs in mouse granulosa cells prior to and $4 \mathrm{~h}$ after human chorionic gonadotropin treatment and further investigation indicated that miR-21 could inhibit apoptosis of mouse periovulatory granulosa cells (9). A previous study demonstrated differential miRNA expression profiles in the plasma of patients with POF and healthy females by miRNA microarray analysis. A total of 10 upregulated miRNAs were identified, including miR-146a (8). Nevertheless, the signaling pathways that regulate the expression of miRNAs during POF and the function of individual miRNAs in granulosa cell apoptosis remain to be elucidated.

Interleukin (IL)-1 receptor-associated kinase (IRAK1) and tumor necrosis factor receptor-associated factor 6 (TRAF6), 
two key adaptor/scaffold proteins in the IL-1 and Toll-like receptor (TLR) signaling pathway, are known to positively regulate nuclear factor $(\mathrm{NF})-\kappa \mathrm{B}$ activity, which is presented by phosphorylation of I $\mathrm{B} \alpha$. IRAK1 and TRAF6 are proposed to be targeted by miR-146a as a part of the NF- $\mathrm{B}$-induced negative feedback loop $(10,11)$. NF- $\kappa \mathrm{B}$ has been demonstrated to be involved in the immune response, inflammation, apoptosis and other biological processes (12). The caspase cascade is an important pathway for apoptosis. Activation of caspase- 8 and caspase-9 initiates cell apoptosis, which causes the executor of cell apoptosis caspase-3 to be cleaved and degrades the substrate poly (ADP-ribose) polymerase (PARP) (13). Thus, it was hypothesized that miR-146a contributes to the apoptosis of ovarian granulosa cells via the caspase cascade by directly targeting IRAK1 and TRAF6.

The present study generated gain-of and loss-of function cell models of miR-146a, and various inhibitors of apoptosis were used. The aim of the study was to explore the functional role of miR-146a in POF and identify the possible molecular mechanism for the modulation of miR-146a in POF.

\section{Materials and methods}

Tissue samples. Six plasma samples from different patients with POF and two age-matched normal plasma samples were obtained from the Xiangya Hospital, Central South University (Changsha, China) and diagnosis was confirmed by routine pathological examination. All experiments were conducted following obtaining approval from the Ethics Committee of Central South University. Each patient provided written informed consent.

Isolation of human granulosa cells from patients with POF. Isolation of human ovarian granulosa cells from follicular fluid was performed as previously described (8). Briefly, all granulosa cells were disaggregated and incubated with $10 \%$ hyaluronidase for $15 \mathrm{~min}$ at $37^{\circ} \mathrm{C}$. Subsequently, percoll (Sigma-Aldrich, St. Louis, MO, USA) was used to separate granulosa cells from red blood cells and lymphocytes by density gradient centrifugation at $1,000 \mathrm{x}$ g for $15 \mathrm{~min}$. The granulosa cells were harvested and cultured in RPMI-1640 medium (Gibco-BRL, Carlsbad, CA, USA) supplemented with $10 \%$ fetal bovine serum and $1 \%(\mathrm{w} / \mathrm{v})$ penicillin/ streptomycin in a $5 \% \mathrm{CO}_{2}$ humidified atmosphere at $37^{\circ} \mathrm{C}$. In the present study, the cells were termed POFC-112 and POFC-38.

Normal human ovarian granulosa cells, COV434, were purchased from the Peking Union Medical College Cell Bank (Beijing, China). The cells were cultured in RPMI-1640 medium (Gibco-BRL) supplemented with $10 \%$ fetal bovine serum and $1 \%(\mathrm{w} / \mathrm{v})$ penicillin/streptomycin in a $5 \% \mathrm{CO}_{2}$ humidified atmosphere at $37^{\circ} \mathrm{C}$.

Cell treatment. To investigate the role of miR-146a in POF, ectopic expression of miR-146a was achieved by transfecting Lv-pre-miR-146a or Lv-anti-miR-146a using Lipofectamine 2000 (Invitrogen Life Technologies, Carlsbad, CA, USA). Pre-miR scrambled negative (pre-scramble) or anti-miR scrambled negative (anti-scramble) was also transfected into the cells and used as a negative control. To analyze the downstream molecule associated with miR-146a, COV434 cells were treated with the caspase-8 inhibitor Z-LEHD-FMK (Sigma-Aldrich) and the caspase-9 inhibitor Z-IEHD-FMK (Sigma-Aldrich) at a concentration of $100 \mathrm{mM}$ (diluted with dimethyl sulfoxide) for $72 \mathrm{~h}$.

Reverse transcription quantitative polymerase chain reaction (RT-qPCR) for miRNA. qPCR for miRNA was performed using IQ SYBR Green Supermix (Bio-Rad Laboratories, Inc., Hercules, CA, USA). Briefly, $3 \mu \mathrm{g}$ of total RNA was reverse transcribed using a specific looped RT primer for each miRNA using a RevertAid First Strand cDNA synthesis kit (Thermo Fisher Scientific, Waltham, MA, USA). The following amplification was performed using IQ SYBR Green Supermix in a CFX connect Real-Time PCR System (Bio-Rad Laboratories, Inc.). $\beta$-actin was used as an internal control. The primers used were as follows: Has-miR-146a, forward TTATTAAGTATCCAGTGCAGGGTCCGAGG and reverse TTGCGGGACATCTAATACTGCCTGGTAATG; $\beta$-actin, sense 5'-AGGGGCCGGACTCGTCATACT-3' and antisense 5'-GGCGGCACCACCATGTACCCT-3'. The cycle threshold value was used to calculate the normalized expression of miR-146a using Bio-Rad CFX Manager 3.1 software (Bio-Rad Laboratories, Inc.).

$q P C R$ for $m R N A$. TRIzol reagent (Invitrogen Life Technologies) was used to extract total RNA according to the manufacturer's instructions. Total RNA $(0.3 \mu \mathrm{g})$ was reverse transcribed using a RevertAid First Strand cDNA synthesis kit (Thermo Fisher Scientific). The following PCR amplification was performed in a Thermal Cycler Dice Real-time system using SYBR Green qPCR mix (Bio-Rad Laboratories, Inc.). For each sample, the relative mRNA level was normalized to $\beta$-actin. The following primer pairs were used: IRAK1, sense 5'-CCTCCACCTTCCTCTCCCCA-3' and anti-sense 5'-CACCGTGTTCCTCATCACCG-3'; TRAF6, sense 5'-AGTGTCTGTGTCCGTCCTCTA-3' and anti-sense 5'-CTTGCTCTGATTCTCTCCTTC-3'; $\beta$-actin, sense 5'-AGGGGCCG GACTCGTCATACT-3' and antisense 5'-GGCGGCACCACCATGTACCCT-3'.

Dual luciferase reporter assay. A wild type 3'-UTR of IRAK1 (wt-IRAK1) and it's mutant type 3'-untranslated region (UTR) of IRAK1 (mut-IRAK1), and a wild type 3'-UTR of TRAF6 (wt-TRAF6) and it's mutant type 3'-UTR of TRAF6 (mut-TRAF6) were constructed into the dual luciferase reporter vector, respectively. For the luciferase assay, $10^{5}$ cells were seeded in 6 -well plates for $12 \mathrm{~h}$. Subsequently, the cells were co-transfected with the indicated dual luciferase reporter vector and miR-146a mimic (pre-miR-146a) or miR-146a inhibitor (anti-miR-146a), or the negative control (pre-scramble and anti-scramble), respectively. Following $5 \mathrm{~h}$ incubation with transfection reagent, the medium was refreshed with fresh completed medium. Following transfection for $48 \mathrm{~h}$, the luciferase activities in each group were measured using a dual luciferase reporter gene assay kit (Promega Corporation, Madison, WI, USA) and detected using an LD400 luminometer (Promega Corporation). Renilla luciferase activity was normalized to firefly luciferase activity. 
A

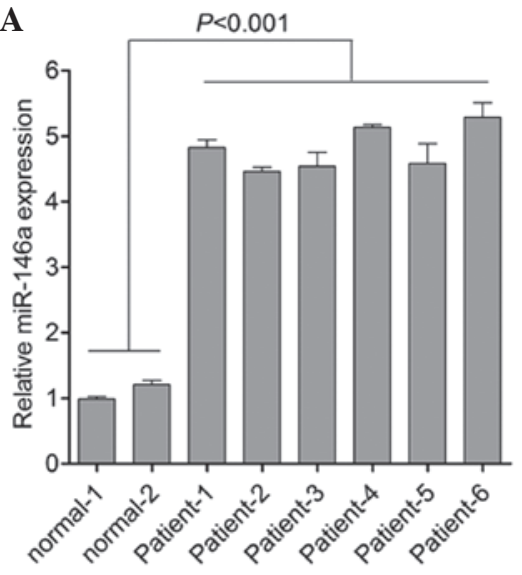

B

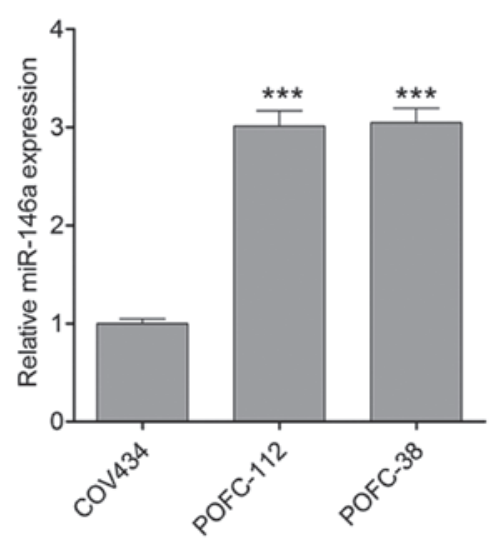

Figure 1. miR-146a is upregulated in plasma and ovarian granulosa cells from patients with POF. (A) qPCR was used to determine the expression of miR-146a in plasma samples. (B) qPCR was used to determine the expression of miR-146a in ovarian granulosa cells from patients with POF. The COV434 cell line was used as a normal control. Data are presented as the mean \pm standard error of the mean. ${ }^{* * *} \mathrm{P}<0.001$ vs. control. POF, premature ovarian failure; qPCR, quantitative polymerase chain reaction; miR-146a, microRNA-146a.

Western blot analysis. Total protein was extracted from the indicated cells using RIPA lysis buffer (Auragene Bioscience Inc., Changsha, China). A BCA Protein Assay kit (Thermo Fisher Scientific) was used to determine the protein concentration. Total proteins $(60 \mu \mathrm{g})$ mixed in loading buffer were denaturalized, separated by SDS-PAGE and transferred onto a polyvinylidene fluoride membrane (Millipore, Billerica, MA, USA). Following incubation with the appropriate primary antibodies at $37^{\circ} \mathrm{C}$ for $3 \mathrm{~h}$, the membrane was washed and incubated with horseradish peroxidase-conjugated secondary antibody. The signal was visualized by the Chemi-Lumi One system (Nacalai Tesque, Kyoto, Japan). Data were analyzed by densitometry using Image-Pro plus software 6.0 (Media Cybernetics, Rockville, MD, USA) and normalized to $\beta$-actin expression. The following primary antibodies (1:1,000 dilution) were purchased from Abcam (Cambridge, MA, USA): Rabbit polyclonal anti-IRAK1 (cat. no. ab190234), rabbit polyclonal anti-TRAF6 (cat. no. ab181622), rabbit polyclonal anti-NFאB-p65 (cat. no. ab16502), rabbit polyclonal anti-pNFkB-p65 (cat. no. ab86299), rabbit polyclonal anti-IKB $\alpha$ (cat. no. ab7217), mouse monoclonal anti-pIKB $\alpha$ (cat. no. ab12135), rabbit polyclonal anti-cleaved caspase 3 (cat. no. ab2302), rabbit monoclonal anti-cleaved PARP (cat. no. ab32064), rabbbit polyclonal anti-caspase-9 (cat. no. ab69514) and rabbit polyclonal anti-caspase-8 (cat. no. ab25901). Mouse monoclonal anti- $\beta$-actin (1:500 dilution; cat. no. YM3028) was purchased from Immunoway (Newark, DE, USA) and horseradish peroxidase-conjugated secondary anti-rabbit IgG (cat. no. 7074S) and anti-mouse IgG (cat.no. 3420S) were obtained from Cell Signaling Technology, Inc. (Danvers, MA, USA).

Apoptosis assay. Cells were transfected with $80 \mathrm{nM}$ miR-146a precursor (pre-miR-146a) or $80 \mathrm{nM}$ miR-146a inhibitor (anti-miR-146a), respectively, and $48 \mathrm{~h}$ after transfection the cells were then subjected to an apoptosis assay. Apoptosis was detected by annexin V/propidium iodide staining with the apoptosis detection kit (BD Biosciences, San Jose, CA, USA). Briefly, $10^{6}$ treated cells were incubated with annexin V/propidium iodide for $20 \mathrm{~min}$ at $25^{\circ} \mathrm{C}$. The cells were then analyzed by flow cytometry.
Terminal deoxynucleotidyl transferase dUTP nick end labeling (TUNEL) assay. A TUNEL assay was also used to detect the apoptosis of COV434 cells transfected with pre-miR-146a or anti-miR-146a. Briefly, the cells were smeared on slides. Following 30 min fixed with $4 \%$ paraformaldehyde at room temperature, the slides were incubated with $0.1 \%$ Triton $\mathrm{X}-100$ for $10 \mathrm{~min}$, rinsed with phosphate-buffered saline (PBS), incubated with $3 \% \mathrm{H}_{2} \mathrm{O}_{2}$ to block endogenous peroxidase activity, and rinsed with PBS. Following that, an apoptosis detection kit (BD Biosciences) was used for TUNEL staining in accordance with the manufacturer's instructions. The TUNEL-stained slides were observed under a fluorescence microscope [AE31; Motic Xiamen (China), Fujian, China].

Statistical analysis. Statistical evaluation for data analysis was determined by unpaired Student's t-test. Data are presented as the mean \pm standard deviation of three independent experiments. Data were analyzed using SPSS 17.0 statistical software (SPSS Inc., Chicago, IL, USA). P<0.05 was considered to indicate a statistically significant difference.

\section{Results}

miR-146a is frequently upregulated in plasma and human granulosa cells from patients with POF. The present study focused on the role of miR-146a in POF. The present study initially detected the expression of miR-146a in peripheral plasma from patients with POF. As shown in Fig. 1A, the results of qPCR for miR-146a in plasma samples demonstrated that compared with the normal control, the expression of miR-146a was significantly upregulated in patients with POF. Furthermore, the expression of miR-146a was also detected in granulosa cells from patients with POF. In accordance with the results from plasma, an increase in miR-146a expression was observed in granulosa cells obtained from patients with POF (Fig. 1B). This result indicated that there was a correlation between miR-146a and POF.

miR-146a regulates cell apoptosis in COV434 cells. To further determine the role of miR-146a in POF, gain- and 
A
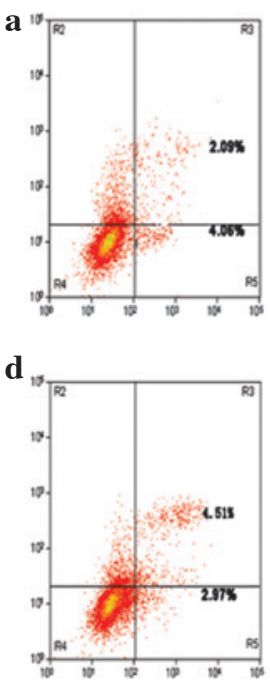

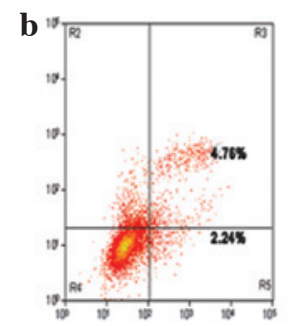

e

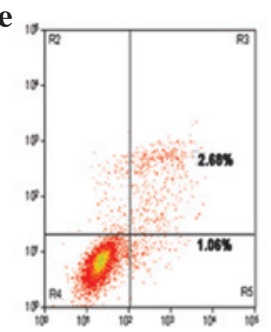

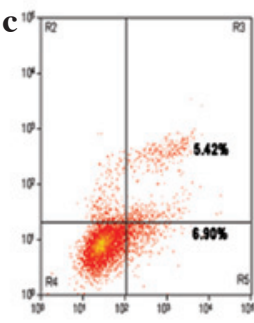

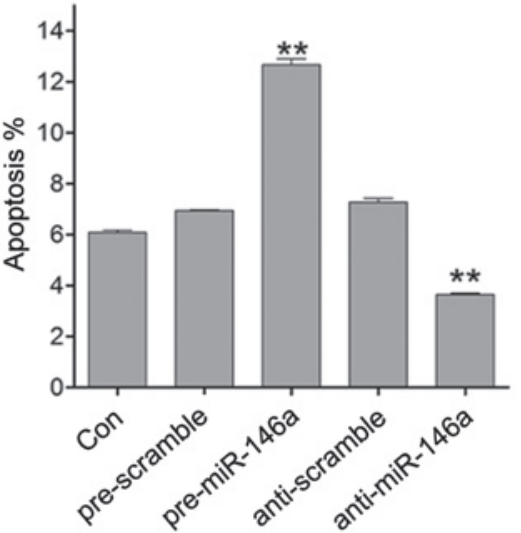

B

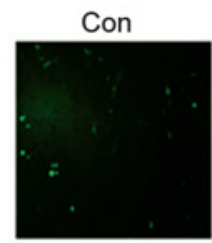

pre-scramble
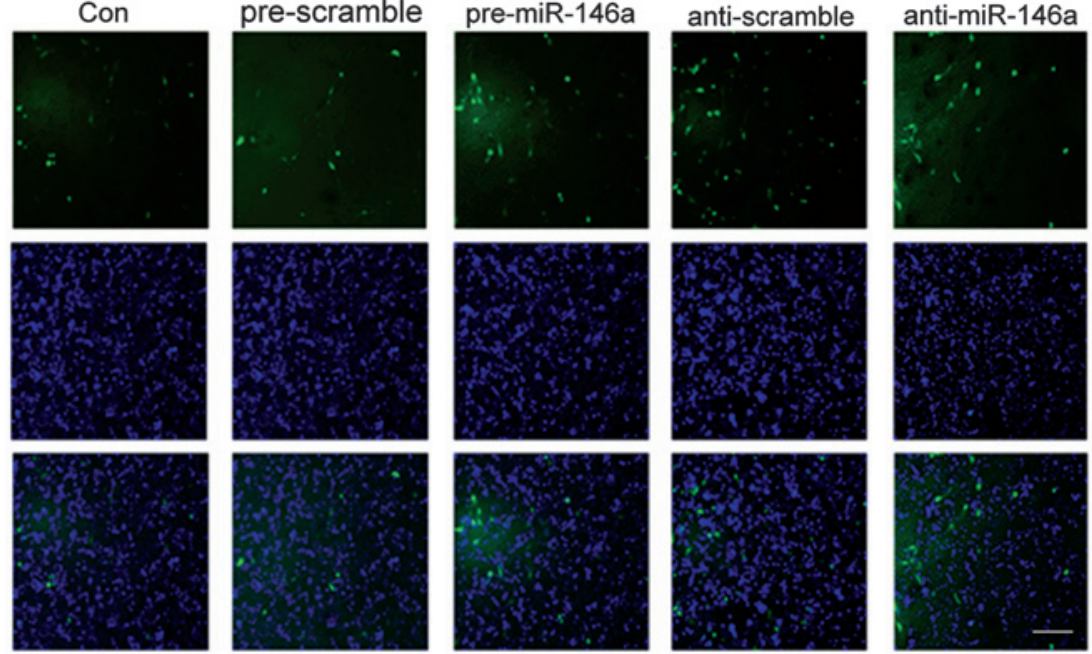

Figure 2. miR-146a contributes to ovarian granulosa cell apoptosis. (A) Cell apoptosis of COV434 cells treated with pre-miR-146a or anti-miR-146a were determined by (a-e) flow cytometric analysis and quantified. (B) Cell apoptosis induced by miR-146a was measured by TUNEL staining. Data are presented as the mean \pm standard error of the mean. ${ }^{* *} \mathrm{P}<0.01$ vs. control. TUNEL, terminal deoxynucleotidyl transferase dUTP nick end labeling; miR-146a, microRNA-146a.

loss-functional experiments were performed by transfecting pre-miR-146a or anti-miR-146a into COV434 cells. The present study examined the apoptosis of COV434 cells following upregulating or downregulating miR-146a by flow cytometry. As shown in Fig. 2A, upregulation of miR-146a promoted cell apoptosis, whereas downregulation of miR-146a reduced cell apoptosis. In addition, a TUNEL assay was used to confirm the results of flow cytometry. Consistent with the results of flow cytometry, it was found that overexpression of miR-146a increased the number of TUNEL-positive cells compared with the negative control, and reducing the expression of miR-146a decreased the number of TUNEL-positive cells compared with the negative control (Fig. 2B). Thus, the data confirmed that miR-146a regulates cell apoptosis in COV434 cells in vitro.

miR-146a directly targets IRAK1 and TRAF6. Since miR-146a is upregulated in patients with POF, the target genes of miR-146a were next examined, as miRNAs must produce their effects through their target genes. Bioinformatics is a useful tool for screening the targets of miRNAs. Using the TargetScan and microRNA website (www.targetscan.org), two putative targets, IRAK1 and TRAF6, of miR-146a were selected. To confirm whether miR-146a directly targeted the 3'UTR of IRAK1 and TRAF6, the 3'UTR of IRAK1 and TRAF6 was cloned downstream to a luciferase reporter gene (wt-IRAK1 and wt-TRAF6). The wt-IRAK1 or wt-TRAF6 vector was co-transfected with the miR-146a mimics or inhibitor into COV434 cells, respectively. The luciferase activity of miR-146a mimic-transfected cells was significantly decreased compared with cells co-transfected with their mutant type and scramble control cells (Fig. 3A and B). The present study then assessed whether miR-146a directly regulates the expression of IRAK1 and TRAF6. qPCR was used to detect the expression of miR-146a following miR-146a mimic or inhibitor treatment. The results demonstrated that miR-146a mimics efficiently induced the expression of miR146a, and the miR-146a inhibitor markedly downregulated the expression of miR-146a (Fig. 3C). Furthermore, miR-146a mimics efficiently reduced the mRNA expression of IRAK1 and TRAF6 whereas the miR-146a inhibitor markedly upregulated the mRNA expression of IRAK1 and TRAF6 (Fig. 3D and E). In addition, the protein expression of IRAK1 and TRAF6 was 
A

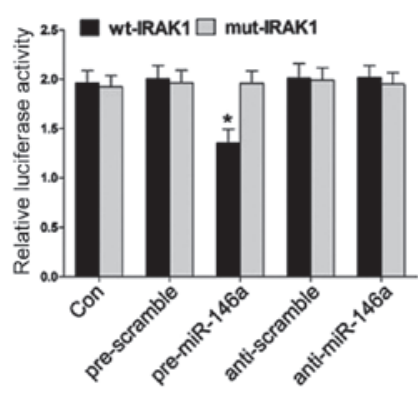

C

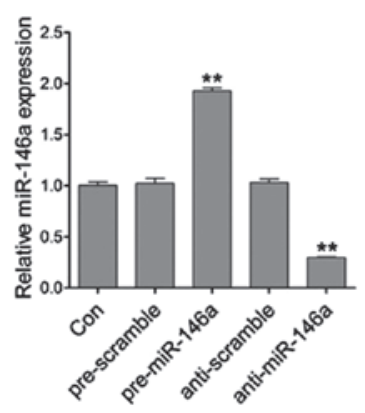

F

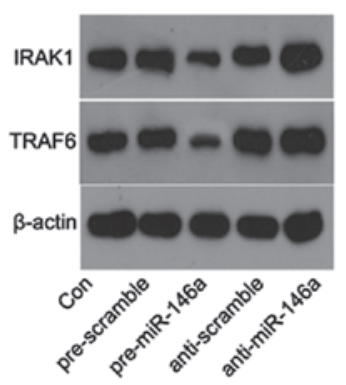

D
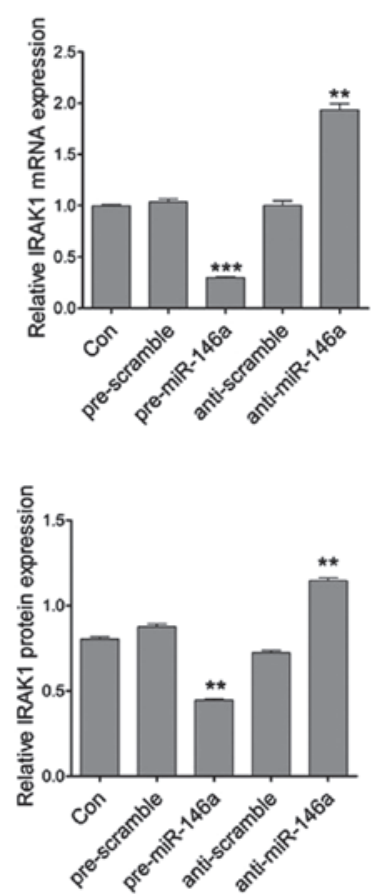

B

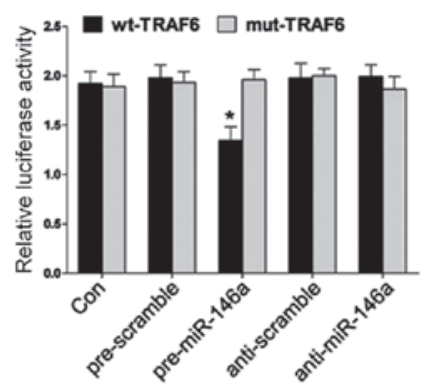

E

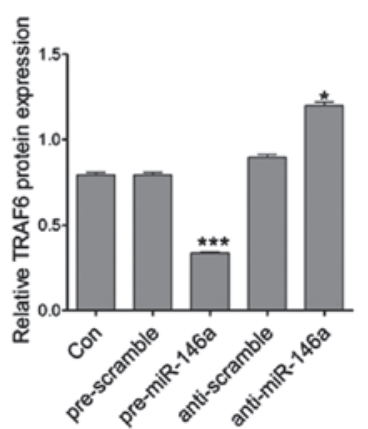

Figure 3. miR-146a simultaneously targets IRAK1 and TRAF6. (A) A dual luciferase reporter gene assay was used to confirm the putative target, IRAK1, of miR-146a. (B) A dual luciferase reporter gene assay was used to confirm the putative target, TRAF6, of miR-146a. (C) Expression of miR-146a was detected by qPCR following transfection of pre-miR-146a or anti-miR-146a. (D) IRAK1 mRNA expression was detected by qPCR in COV434 cells treated with pre-miR-146a or anti-miR-146a. (E) TRAF6 mRNA expression was detected by qPCR in COV434 cells treated with pre-miR-146a or anti-miR-146a. (F) IRAK1 and TRAF6 protein expression was detected by western blot analysis and quantified in COV434 cells treated with pre-miR-146a or anti-miR-146a. Data are presented as the mean \pm standard error of the mean. ${ }^{*} \mathrm{P}<0.05,{ }^{* * *} \mathrm{P}<0.01$ and ${ }^{* * * * *} \mathrm{P}<0.001$ vs. control. miR-146a, microRNA146a; IRAK1, interleukin- 1 receptor-associated kinase; TRAF6, tumor necrosis factor receptor-associated factor 6; qPCR, quantitative polymerase chain reaction.

further analyzed in cells transfected with the miR-146a mimics or inhibitor. Similarly, the protein expression of IRAK1 and TRAF6 was decreased or induced by the miR-146a mimics or inhibitor, respectively (Fig. 3F). These results suggested that miR-146a regulated IRAK1 and TRAF6 expression at the transcriptional and translational levels.

miR-146a regulates the expression of $p N F \kappa B-p 65, p I \kappa B \alpha$, caspase-8, caspase-9, cleaved caspase-3 and cleaved PARP. To examine the mechanism underlying the regulation of cell apoptosis by miR-146a in COV434 cells, the downstream target genes associated with apoptosis of COV434 cells were detected. Considering the role of miR-146a in cell apoptosis, the molecules involved in apoptosis were detected in granulosa cells. The expression levels of pNFkB-p65, pI $\mathrm{B} \alpha$, caspase- 8 , caspase- 9 , cleaved caspase- 3 and cleaved PARP were measured by western blotting in pre-miR-146a or anti-miR-146a-transfected COV434 and negative control cells. As shown in Fig. 4A, the expression of pNFkB-p65 and $\mathrm{pI \kappa \textrm {B }} \alpha$ was reduced following pre-miR-146a transfection at the protein level, whereas it was markedly induced by anti-miR-146a transfection compared with the negative control. However, upregulation and downregulation of miR-146a did not alter the expression of NFкB-p65 and IкB $\alpha$. Caspase signaling is important for apoptosis. Thus, the protein levels of caspase-8, caspase-9, cleaved caspase- 3 and cleaved PARP were next detected by western blotting in pre-miR-146a or anti-miR-146a-transfected COV434 and control cells. Compared with the negative control, the protein level of caspase-8, caspase- 9 , cleaved caspase- 3 and cleaved PARP was significantly increased in pre-miR-146a-transfected cells, whereas it was markedly reduced in anti-miR-146a-transfected cells (Fig. 4B). These results indicated that miR-146a contributed to granulosa cell apoptosis.

miR-146a regulates cell apoptosis via caspase signaling. To further determine the signaling pathway of miR-146a contributing to cell apoptosis in granulosa cells, the caspase pathway 

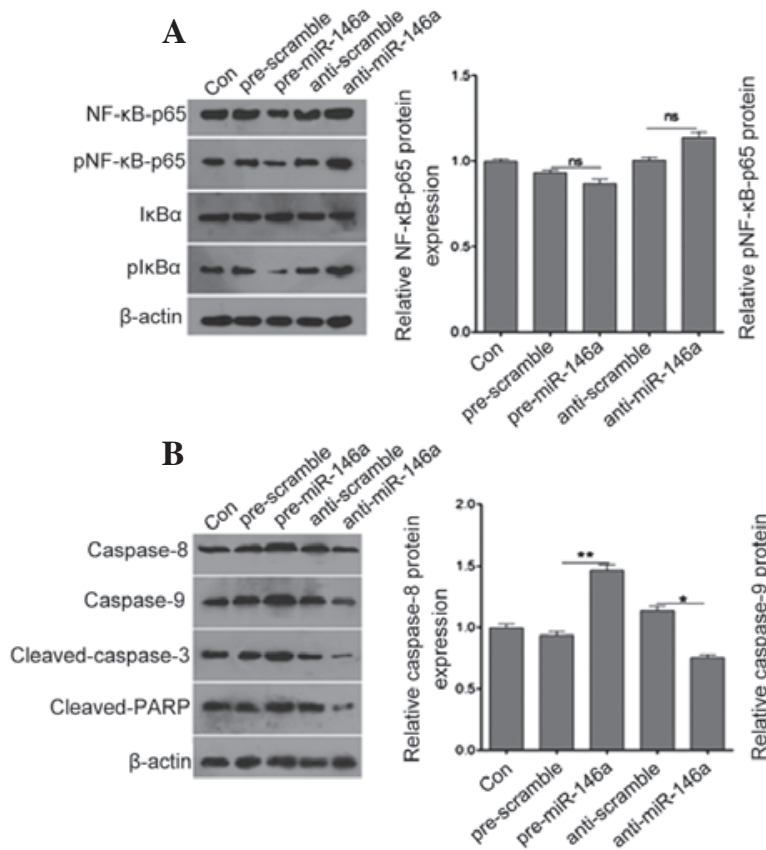
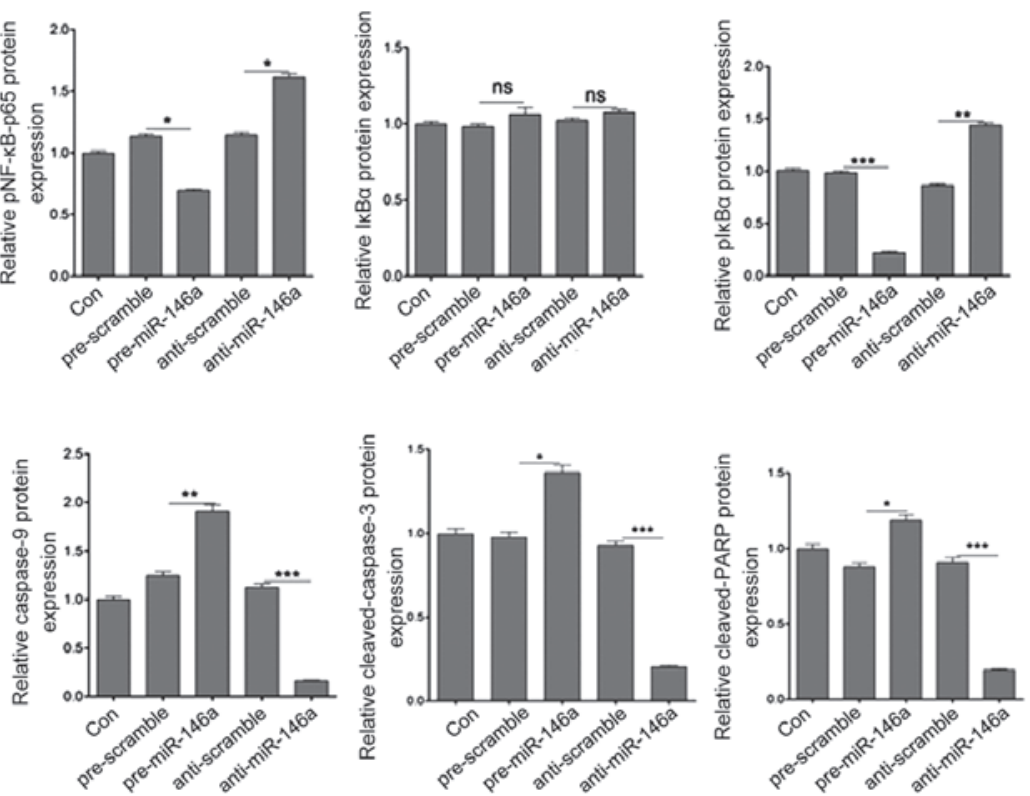

Figure 4. miR-146a regulates the expression of pNFкB-p65, pIкB $\alpha$, caspase- 8 , caspase-9, cleaved caspase-3 and cleaved PARP. (A) Western blot analysis was

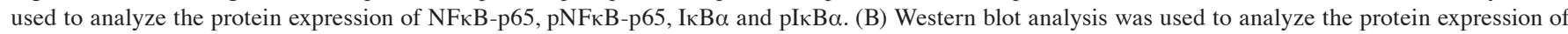
caspase-8, caspase-9, cleaved caspase- 3 and cleaved PARP. Data are presented as the mean \pm standard error of the mean. ${ }^{*} \mathrm{P}<0.05,{ }^{* *} \mathrm{P}<0.01$ and ${ }^{* * * *} \mathrm{P}<0.001$ vs.

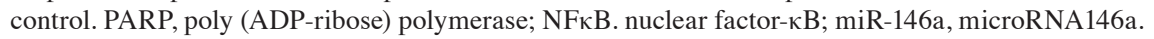

A a

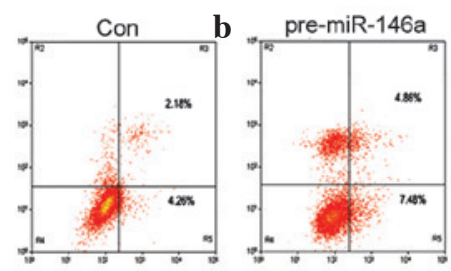

d
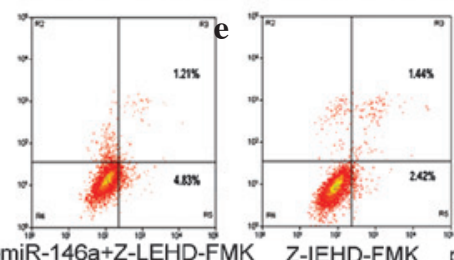

pre-miR-146a+Z-LEHD-FMK Z-IEHD-FMK pre-miR-146̈a+Z̈-IEHD-FMK
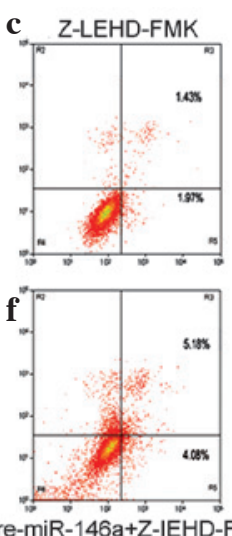

B

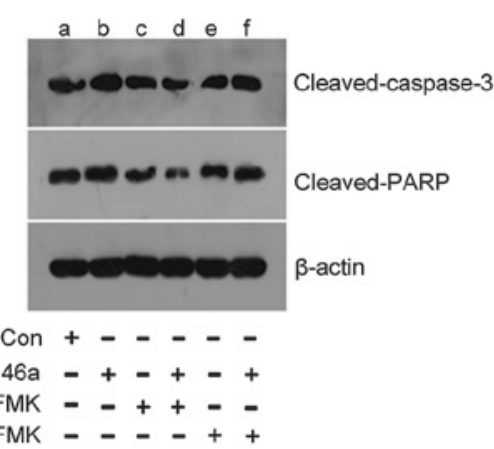

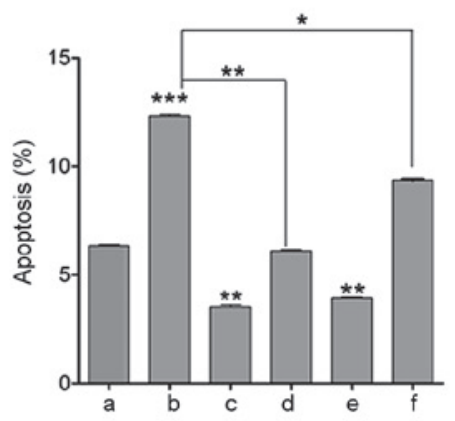
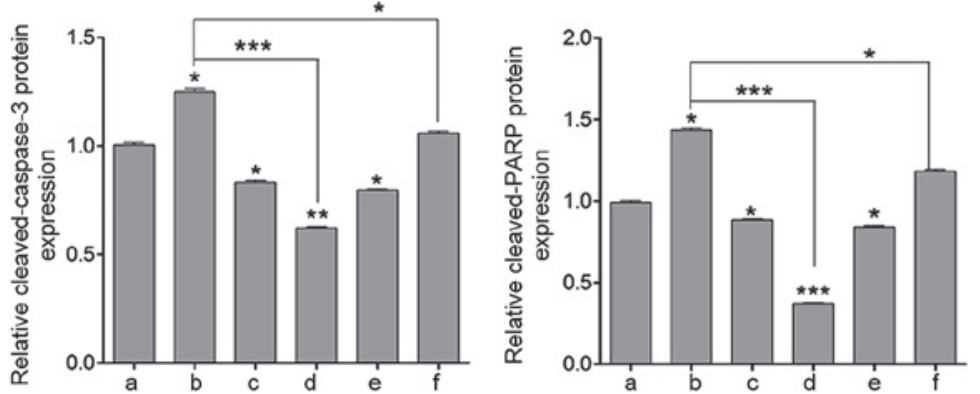

Figure 5. miR-146a regulates cell apoptosis via caspase signaling. (A) Cell apoptosis was determined by (a-f) flow cytometric analysis and quantified. (B) Western blot analysis was used to analyze the protein expression of cleaved caspase-3 and cleaved PARP. Data are presented as the mean \pm standard error of the mean. ${ }^{*} \mathrm{P}<0.05,{ }^{* *} \mathrm{P}<0.01$ and ${ }^{* * *} \mathrm{P}<0.001$ vs. control. miR-146a, microRNA146a; PARP, poly (ADP-ribose) polymerase.

was inhibited using a caspase-8 inhibitor (Z-LEHD-FMK) and caspase-9 inhibitor (Z-IEHD-FMK) following pre-miR-146a or anti-miR-146a treatment. As shown in Fig. 5A, inhibition of caspase- 8 and caspase- 9 by their specific inhibitor significantly attenuated the effect of miR-146a on apoptosis. In addition, gene expression associated with apoptosis was detected, 
including cleaved caspase- 3 and cleaved PARP by western blot analysis. As shown in Fig. 5B, the protein expression of cleaved caspase- 3 and cleaved PARP was induced by upregulating miR-146a and decreased by the caspase- 8 inhibitor and caspase-9 inhibitor. When co-treating the caspase inhibitor with pre-miR-146a, it was found that the increase in cleaved caspase- 3 and cleaved PARP expression was decreased by the caspase- 8 inhibitor and caspase- 9 inhibitor. Thus, these results indicated that miR-146a contributed to granulosa cell apoptosis via caspase signaling.

\section{Discussion}

Although the causes of POF remain to be elucidated, POF is characterized by increased apoptosis of ovarian granulosa cells (14). Increasing evidence has demonstrated that miRNAs are involved in multiple cell processes, including survival, proliferation and apoptosis (15). Certain differentially expressed miRNAs in the plasma of POF patients have been identified, including downregulated miRNAs (let-7c and miR-144) and upregulated miRNAs (miR-202 and miR-146a) (8). Numerous previous studies have demonstrated that miR-146a is implicated in cell apoptosis. It was reported that upregulation of miR-146a induced apoptosis of human chondrocytes (16) and miRNA-146a regulated the maturation and differentiation of vascular smooth muscle cells (17). In addition, miR-146a induces apoptosis in several types of cancer, including non-small cell lung cancer (18), gastric cancer (19) and breast cancer (20). However, the role of miR-146a in POF remains to be elucidated. In the present study, in line with a previous study (8), it was confirmed that miR-146a was significantly upregulated in the plasma of POF patients compared with the normal control. Furthermore, in isolated ovarian granulosa cells from patients with POF, the expression of miR-146a was markedly increased. Thus, these results indicated that miR-146a is important in the apoptosis of ovarian granulosa cells in patients with POF.

By gain- and loss- of function analyses, it was found that upregulation of miR-146a induced apoptosis of ovarian granulosa cells, whereas downregulation of miR-146a reduced apoptosis of ovarian granulosa cells. miR-146a functions as an apoptosis regulator by targeting the 3 'UTR of target genes. Using bioinformatic tools, two putative targets of miR-146a were found, including IRAK1 and TRAF6. In addition, by using the dual luciferase reporter assay, it was confirmed that miR-146a directly targeted the 3'UTR of IRAK1 and TRAF6 in ovarian granulosa cells. In addition, miR-146a regulated the expression of IRAK1 and TRAF6 at the transcriptional and translational levels in ovarian granulosa cells. TLR signaling within ovarian granulosa cells has broad implications for ovarian physiology. Woods et al demonstrated that treatment with lipopolysaccharide in vitro led to the differential regulation of TLRs based on the stage of follicle maturation (21), suggesting that follicle maturation was required for the TLR signaling gene. IRAK-1 and TRAF-6 are two key elements involved in the TLR signaling pathway. It was reported that miR-146a modulated inflammatory responses by targeting IRAK1 and TRAF6 in macrophages (22). miR-146a has been demonstrated as a regulator of the inflammatory response $(23,24)$. Thus, the present study provided evidence to demonstrate that miR-146a contributes to the apoptosis of ovarian granulosa cells via TLR signaling.

IRAK-1 acts as the essential upstream adaptor that mediates signaling to $\mathrm{NF}-\kappa \mathrm{B}$ through the TRAF6 cascade (25). The results from the present study demonstrated that upregulation of miR-146a reduced activation of $N F-\kappa B$ and $\mathrm{I} \kappa \mathrm{B} \alpha$ through targeting IRAK1 and TRAF6. TLR signaling is closely associated with the caspase cascade. It was reported that activated TLR signaling in microglia was inhibited by the pan caspase inhibitor zVAD-fmk and the caspase- 8 inhibitor IETD-fmk (26). TLR3 stimulation induced activation of apoptotic caspases $-8,-9$ and -3 (27). In addition, TLR4 induces the assembly of caspase-8-based signaling complexes (28). The present study demonstrated that ectopic expression of miR-146a affects the caspase cascade, which alters the expression of caspase-8, caspase-9, cleaved caspase-3 and cleaved PARP. By inhibiting caspase- 8 and caspase- 9 using their specific inhibitors, it was found that apoptosis induced by miR-146a was attenuated by caspase inhibitors. In addition, when co-treated with the caspase inhibitor, the role of miR-146a in promoting apoptosis was inhibited. Thus, it was demonstrated that miR-146a mediated ovarian granulosa cell apoptosis via caspase signaling.

In conclusion, upregulation of miR-146a in the plasma and ovarian granulosa cells of patients with POF may act as a biomarker for POF. Furthermore, miR-146a regulated TLR signaling by simultaneously targeting IRAK1 and TRAF6, which affected the activity of $\mathrm{NF}-\kappa \mathrm{B}$ and $\mathrm{I} \kappa \mathrm{B} \alpha$, and the caspase cascade. Thus, miR-146a/IRAK1/TRAF6/caspase- 8 signaling mediated apoptosis of ovarian granulosa cells in POF, suggesting that inhibition of miR-146a may be beneficial for the management of POF.

\section{References}

1. Beck-Peccoz P and Persani L: Premature ovarian failure. Orphanet J Rare Dis 1: 9, 2006.

2. Mittal M, Savvas M, Arya R, McEniery C, Narvekar N, Cardozo L, Panay N and Hamoda H: A randomised controlled trial comparing the effects of micronized progesterone to medroxyprogesterone acetate on cardiovascular health, lipid metabolism and the coagulation cascade in women with premature ovarian insufficiency: Study protocol and review of the literature. Menopause Int 19: 127-132, 2013.

3. Morgan S, Anderson RA, Gourley C, Wallace WH and Spears N: How do chemotherapeutic agents damage the ovary? Hum Reprod Update 18: 525-535, 2012.

4. Knet M, Tabarowski Z, Slomczynska M and Duda M: The effects of the environmental antiandrogen vinclozolin on the induction of granulosa cell apoptosis during follicular atresia in pigs. Theriogenology 81: 1239-1247, 2014.

5. Ford JH: Reduced quality and accelerated follicle loss with female reproductive aging - does decline in theca dehydroepiandrosterone (DHEA) underlie the problem? J Biomed Sci 20: 93, 2013.

6. Sirotkin AV, Lauková M, Ovcharenko D, Brenaut $\mathrm{P}$ and Mlyncek M: Identification of microRNAs controlling human ovarian cell proliferation and apoptosis. J Cell Physiol 223: 49-56, 2010.

7. Toloubeydokhti T, Bukulmez O and Chegini N: Potential regulatory functions of microRNAs in the ovary. Semin Reprod Med 26: 469-478, 2008.

8. Yang X, Zhou Y, Peng S, Wu L, Lin HY, Wang S and Wang H: Differentially expressed plasma microRNAs in premature ovarian failure patients and the potential regulatory function of mir-23a in granulosa cell apoptosis. Reproduction 144: 235-244, 2012.

9. Fiedler SD, Carletti MZ, Hong X and Christenson LK: Hormonal regulation of MicroRNA expression in periovulatory mouse mural granulosa cells. Biol Reprod 79: 1030-1037, 2008. 
10. Zilahi E, Tarr T, Papp G, Griger Z, Sipka S and Zeher M: Increased microRNA-146a/b, TRAF6 gene and decreased IRAK1 gene expressions in the peripheral mononuclear cells of patients with Sjögren's syndrome. Immunol Lett 141: 165-168, 2012.

11. Hung PS, Liu CJ, Chou CS, Kao SY, Yang CC, Chang KW, Chiu TH and Lin SC: miR-146a enhances the oncogenicity of oral carcinoma by concomitant targeting of the IRAK1, TRAF6 and NUMB genes. PLoS One 8: e79926, 2013.

12. Woods DC, White YA, Dau C and Johnson AL: TLR4 activates NF- $\kappa$ B in human ovarian granulosa tumor cells. Biochem Biophys Res Commun 409: 675-680, 2011.

13. Park EJ, Shin JW, Seo YS, Kim DW, Hong SY, Park WI and Kang BM: Gonadotropin-releasing hormone-agonist induces apoptosis of human granulosa-luteal cells via caspase- $8,-9$ and -3 and poly-(ADP-ribose)-polymerase cleavage. Biosci Trends 5: 120-128, 2011.

14. Ebrahimi M and Akbari Asbagh F: Pathogenesis and causes of premature ovarian failure: An update. Int J Fertil Steril 5: 54-65, 2011.

15. Kim YJ, Ku SY, Kim YY, Liu HC, Chi SW, Kim SH, Choi YM Kim JG and Moon SY: MicroRNAs transfected into granulosa cells may regulate oocyte meiotic competence during in vitro maturation of mouse follicles. Hum Reprod 28: 3050-3061, 2013

16. Jin L, Zhao J, Jing W, Yan S, Wang X, Xiao C and Ma B: Role of miR-146a in human chondrocyte apoptosis in response to mechanical pressure injury in vitro. Int J Mol Med 34: 451-463, 2014.

17. Dong S, Xiong W, Yuan J, Li J, Liu J and Xu X: MiRNA-146a regulates the maturation and differentiation of vascular smooth muscle cells by targeting NF- $\kappa$ B expression. Mol Med Rep 8 407-412, 2013.

18. Chen G, Umelo IA, Lv S, Teugels E, Fostier K, Kronenberger P, Dewaele A, Sadones J, Geers C and De Grève J: miR-146a inhibits cell growth, cell migration and induces apoptosis in non-small cell lung cancer cells. PLoS One 8: e60317, 2013.
19. Sha M, Ye J, Zhang LX, Luan ZY and Chen YB: Celastrol induces apoptosis of gastric cancer cells by miR-146a inhibition of NF- $\kappa$ B activity. Cancer Cell Int 13: 50, 2013.

20. Elsarraj HS, Stecklein SR, Valdez K and Behbod F: Emerging functions of microRNA-146a/b in development and breast cancer: MicroRNA-146a/b in development and breast cancer. J Mammary Gland Biol Neoplasia 17: 79-87, 2012.

21. Woods DC, Schorey JS and Johnson AL: Toll-like receptor signaling in hen ovarian granulosa cells is dependent on stage of follicle maturation. Reproduction 137: 987-996, 2009.

22. Li S, Yue Y, Xu W and Xiong S: MicroRNA-146a represses mycobacteria-induced inflammatory response and facilitates bacterial replication via targeting IRAK-1 and TRAF-6. PLoS One 8: e81438, 2013.

23. Liu M, John CM and Jarvis GA: Induction of endotoxin tolerance by pathogenic Neisseria is correlated with the inflammatory potential of lipooligosaccharides and regulated by microRNA-146a. J Immunol 192: 1768-1777, 2014.

24. Quinn EM, Wang JH, O'Callaghan G and Redmond HP: MicroRNA-146a is upregulated by and negatively regulates TLR2 signaling. PLoS One 8: e62232, 2013.

25. Dong W, Liu Y, Peng J, Chen L, Zou T, Xiao H, Liu Z, Li W, Bu Y and Qi Y: The IRAK-1-BCL10-MALT1-TRAF6-TAK1 cascade mediates signaling to NF-kappaB from Toll-like receptor 4. J Biol Chem 281: 26029-26040, 2006.

26. Kim SJ and Li J: Caspase blockade induces RIP3-mediated programmed necrosis in Toll-like receptor-activated microglia. Cell Death Dis 4: e716, 2013.

27. Grimstad $\varnothing$, Husebye $H$ and Espevik T: TLR3 mediates release of IL- $1 \beta$ and cell death in keratinocytes in a caspase- 4 dependent manner. J Dermatol Sci 72: 45-53, 2013.

28. Antonopoulos C, El Sanadi C, Kaiser WJ, Mocarski ES and Dubyak GR: Proapoptotic chemotherapeutic drugs induce noncanonical processing and release of IL- $1 \beta$ via caspase- 8 in dendritic cells. J Immunol 191: 4789-4803, 2013. 